Annls limnol. 9 (1) $1973: 45-61$.

\title{
CYCLE BIOLOGIQUE ET PRODUCTION DE SIALIS LUTARIA L. (MEGALOPTERA) DANS LE LAC DE PORT-BIELH (PYRENÉES CENTRALES)
}

\author{
par N. Giani ${ }^{1}$ et H. Laville ${ }^{1}$.
}

\begin{abstract}
Une population de Sialis lutaria a été étudiée de 1969 à 1971 dans le lac de Port-Bielh (2 $285 \mathrm{~m}$, Pyrénées-Centrales). Dix stades larvaires ont été dénombrés. Le cycle de développement dure trois années.

Les larves se nourrissent essentiellement de crustacés benthiques pendant la première année, de chironomides et d'oligochètes les années suivantes. La croissance larvaire s'effectue pendant l'isothermie d'automne. 66,7 \% de l'accroissement intervient au cours de la troisième année. Le rapport PS/PF varie au cours de la croissance.

La production annuelle nette atteint $5,12 \mathrm{~kg} / \mathrm{ha} / \mathrm{PS}$. Elle représente les $2 / 3$ de celle du chironomide dominant du lac. Le rapport $\mathrm{P} / \mathrm{B} \max =1,34$ montre une certaine stabilité; il est voisin de ceux précédemment trouvés pour deux chironomides du même lac.
\end{abstract}

\section{The life cycle and production of Sialis lutaria L. (Megaloptera) in lake Port-Bielh (Central Pyrenees).}

A population of Sialis lutaria has been studied from 1969 to 1971 in lake Port-Bielh (2 $285 \mathrm{~m}$, central Pyrenees). There are ten larval instars. The whole life cycle takes three years.

Benthic crustaceans are the chief food of larvae in their first year, chironomids and oligochaetes in their second and third years. Larval growth takes place at the time of autumn isothermy. $66,7 \%$ of the increase in weight occurs during the third year. The ratio dry weight/wet weight (PS/PF) varies during the growth periods.

Ratio $P / B \max =\mathbf{1 . 3 4}$ is fairly stable, and is close to values previously determined for two chironomids in the same lake.

Sialis lutaria L., la plus répandue des trois espèces européennes du genre Sialis, colonise des eaux de différents degrés de trophie (Thienemann 1925). Lestage $(1918,1920)$ a donné une bonne des-

1. Laboratoire d’Hydrobiologie, Université Paul-Sabatier, 118, route de Narbonne, 31077 Toulouse Cedex. 
cription de la morphologie de la larve et de la larvule et détaillé le mécanisme de la ponte chez la femelle. Dubois et Geigy (1935) lui ont consacré une importante monographie, abordant plus particulièrement la biologie de sa reproduction, sa métamorphose et son écologie dans un lac suisse de basse altitude, le Sempachersee $(507 \mathrm{~m})$.

Cette espèce est fréquente dans les lacs et mares des Pyrénées (Bertrand 1954). L'étude quantitative du peuplement benthique du lac de Port-Bielh $(2285 \mathrm{~m})$ a permis d'évaluer son abondance relative à $3 \%$ et sa biomasse à $9,4 \%$ de celle des organismes macrobenthiques de la zone "littori-profonde» (Laville 1971); dans la zone «littorale $(0-7 \mathrm{~m})$ son abondance a été estimée à $9,2 \%$ en 1970 .

Dans le cadre de l'étude de la production benthique du lac de Port-Bielh, nous nous sommes proposés de préciser le mode de vie de Sialis lutaria et son rôle dans le fonctionnement de l'écosystème. Le cycle biologique, la croissance pondérale au cours du développement, la dynamique de population, la nutrition des divers stades larvaires et la prédation par les truites sont tour à tour abordés dans ce travail.

\section{1. - MÉTHODES}

60 prélèvements à la drague Ekman ont été réalisés au hasard dans la zone de vase comprise entre 6 et $15 \mathrm{~m}$ de profondeur du 31 janvier 1969 au 9 octobre 1970 , avec une fréquence plus rapprochée de juillet à octobre, pendant la période de dégel.

Du 31 janvier au 27 octobre 1969, 46 récoltes à la drague Petersen ont été effectuées dans les zones de sédiments accumulés entre les blocs granitiques de la zone littorale.

Les premiers échantillons ont été lavés sur place à travers un filet à vide de mailles de $280 \mu$ capable de retenir les larves dont la largeur de la capsule céphalique est supérieure à $300 \mu$ (stade II inclus).

Les dragages Petersen, pour leur part, ont été nettoyés sur un filet à vide de mailles de $280 \mu$ capable de retenir les larves dont sans exception.

Le tri des animaux a été réalisé à la loupe binoculaire, de préférence sur du matériel vivant, sinon après fixation au formol à $4 \%$.

\section{2. - CYCLE BIOLOGIQUE}

Il est possible de distinguer les divers stades des larves d'insectes aquatiques par les mensurations de leur capsule céphalique. 


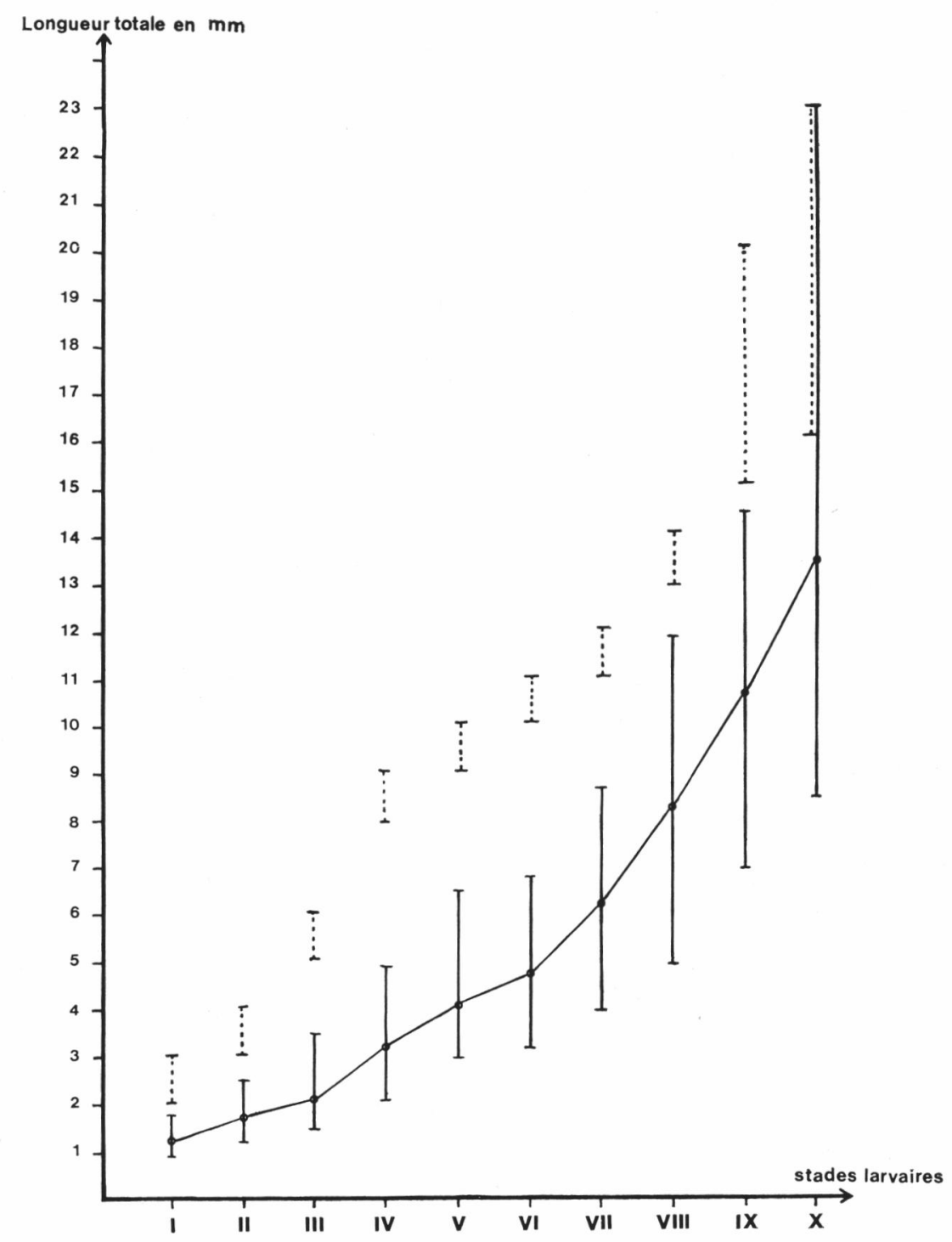

Fig. 1. - Amplitude des longueurs des 10 stades larvaires de Sialis lutaria du lac de Port-Bielh (trait plein) et du Sempachersee (tirets : d'après Dubois et Geigy 1935).

Ce critière s'avère plus précis que celui de la longueur du corps utilisé par Dubois et Geigy (1935); celle-ci présente d'importantes variations au sein de chaque stade, liées au dimorphisme sexuel (fig. 1). 


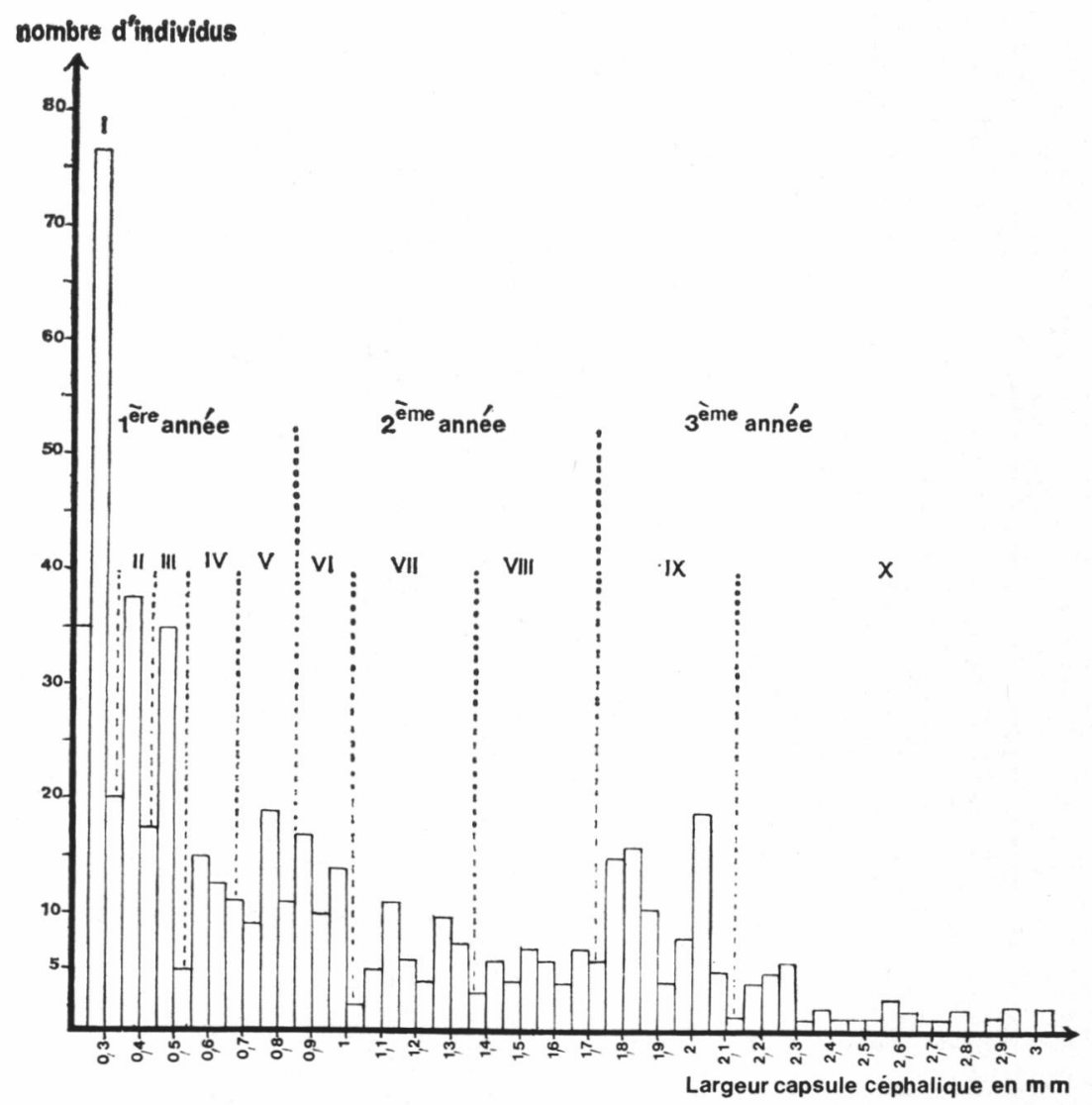

FIg. 2. - Détermination des stades larvaires chez Sialis Iutaria par les mesures de la largeur de la capsule céphalique.

Ce dimorphisme sexuel apparaît sur les diagrammes de la figure 2 au niveau du stade VI; il se manifeste notamment par la taille plus grande des larves femelles.

La mesure de la capsule céphalique révèle la succession des dix stades larvaires déjà dénombrés par Dubois et Geigy (loc. cit.). La longueur des larves des populations du lac de Port-Bielh est toujours supérieure de 2 à $4 \mathrm{~mm}$ à celles du Sempachersee (fig. 1). Les cinq premiers stades, les mieux individualisés, sont unimodaux; à partir du stade VI, le dimorphisme sexuel rend plus délicate la délimitation des stades suivants (fig. 2).

Pour déterminer le cycle biologique de Sialis lutaria, nous avons mesuré la totalité des larves récoltées à diverses profondeurs entre le 30 janvier 1969 et le 9 octobre 1970. Nous avons ainsi obtenu une image de la composition de la population à quatorze dates (fig. 3). 


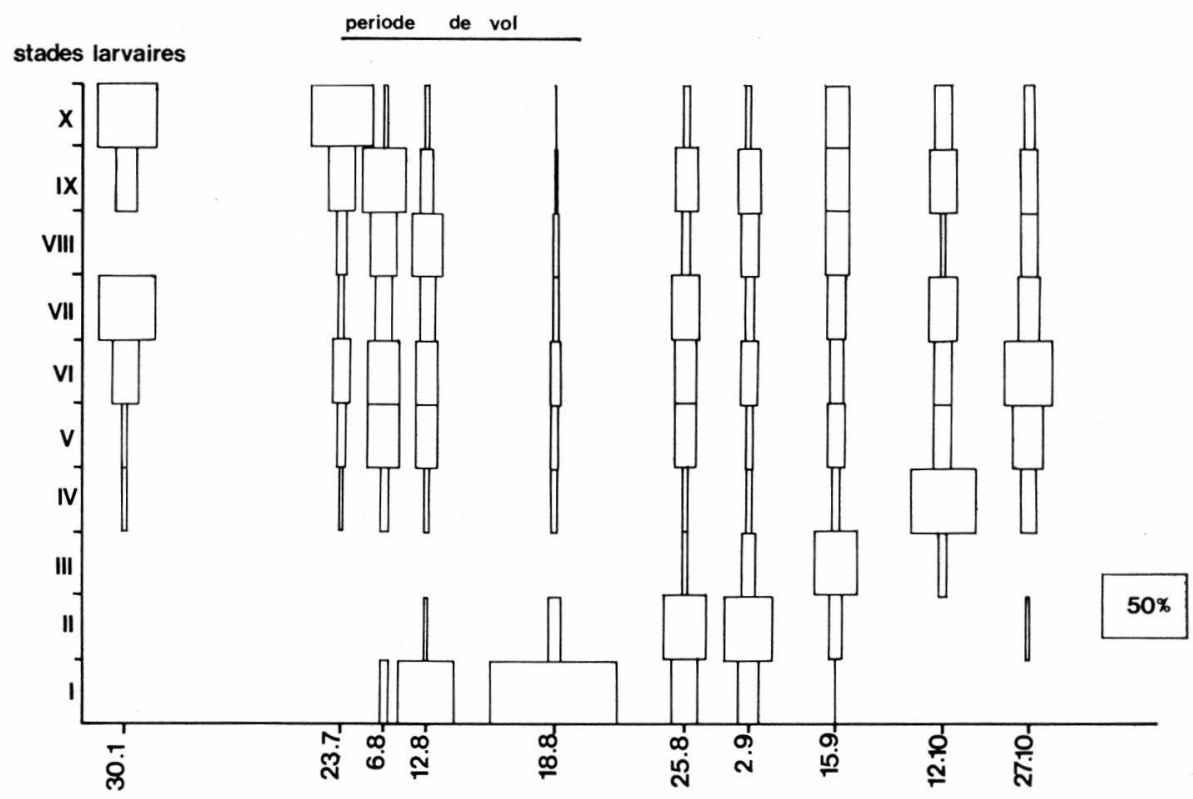

Fig. 3. - Abondance des individus aux différents stades à quatorze dates de prélèvements.

L'évolution de la population montre le plus souvent l'existence simultanée de trois cohortes ou groupes de larves. Une cohorte d'individus de petite taille (stades II à V), une cohorte d'individus de taille moyenne (stades VI à VIII) et une cohorte d'individus de grande taille (stades IX à X). La première cohorte correspond à des larves de première année, la deuxième aux larves de deuxième année, la troisième aux larves de troisième année en fin de cycle de développement. La période d'éclosion des imagos, capturés en vol autour du lac, dure environ cinq semaines, de la mi-juillet à la mi-août. Après fécondation, la ponte des femelles a lieu sur les rochers ou, le plus souvent, sur les tiges de Rhododendron ferrugineum qui surplombent les rives du lac. Les pontes de plusieurs femelles sont généralement rassemblées sur un même support.

L'éclosion des œufs débute au milieu du mois d'août. Après les éclosions, le développement larvaire est rapide; la plupart des larves effectuent quatre mues avant le gel qu'elles atteignent au stade V. Elles demeurent à ce stade pendant tout l'hiver. Au cours du deuxième été, les larves effectuent trois mues pour se trouver au mois d'octobre au stade VIII auquel elles passeront leur deuxième hiver. Les deux dernières mues larvaires interviendront 
au cours du troisième été mais les larves attendront le début de l'été suivant avant d'effectuer leur mue nymphale. La nymphose, de courte durée (environ une semaine), se passe hors de l'eau sur les rives du lac.

Dans le Sempachersee, Dubois et Geigy (loc. cit.) ont mis en évidence un cycle sur deux années. Les larves écloses en mai parviennent au stade VII au mois de janvier; elles effectuent leurs trois dernières mues entre les mois de mars et de septembre. La mue nymphale se situe fin avril - début mai de la deuxième année.

Avec de meilleures conditions thermiques et une nourriture suffisante Sialis lutaria peut vraisemblablement boucler son cycle de développement en une année, si on se réfère à l'espèce néarctique Sialis rotunda Banks étudiée par Azam et Anderson (1969) dans un étang peu profond $(0,65 \mathrm{~m})$ de l'Orégon. Les températures de cet étang sont seulement inférieures à $5{ }^{\circ} \mathrm{C}$ de novembre à janvier et peuvent atteindre jusqu'à $18{ }^{\circ} \mathrm{C}$ en juillet-août. Dans ces conditions, l'espèce acquiert en une année un poids de $68 \mathrm{mg}$ PF comparable au poids moyen (61 $\mathrm{mg}$ PF) atteint en fin de cycle par S. lutaria au lac de Port-Bielh.

Migration de la larve au cours du développement.

Un déplacement des larves de Sialis lutaria a déjà été observé dans le Sempachersee où elles peuvent migrer jusqu'à $20 \mathrm{~m}$ de profondeur; les larves de première année vivent entre 6 et $12 \mathrm{~m}$, celles de deuxième année évoluant entre 12 et $18 \mathrm{~m}$ (Dubois et Geigy 1935).

Au lac de Windermere, elles ne descendent pas au-delà de $12 \mathrm{~m}$ de profondeur (Macan and Worthington 1968).

$\mathrm{Au}$ lac de Port-Bielh, où les larves sont très rarement récoltées au-delà de $15 \mathrm{~m}$, une migration comparable a été mise en évidence.

Nous avons représenté par des courbes l'abondance relative moyenne des stades II, V, VIII, $\mathrm{X}$ aux différentes profondeurs (fig. 4). Ces courbes donnent une image de la répartition bathymétrique des larves et font ressortir deux phénomènes :

- une migration en profondeur des larves au cours de la première année du cycle. Les larves au stade II présentent un net maximum entre 0 et $2 \mathrm{~m}$ ( $80 \%$ de la cohorte) et sont encore peu éloignées des rives où éclosent les larvules. Les larves au stade V ainsi que celles au stade VIII dominent à $9 \mathrm{~m}$ de profondeur.

- une migration en sens inverse des larves de troisième année; le maximum des stades $\mathrm{X}$ se trouvant à $8 \mathrm{~m}$ dénote leur tendance à regagner le bord pour effectuer leur nymphose. 


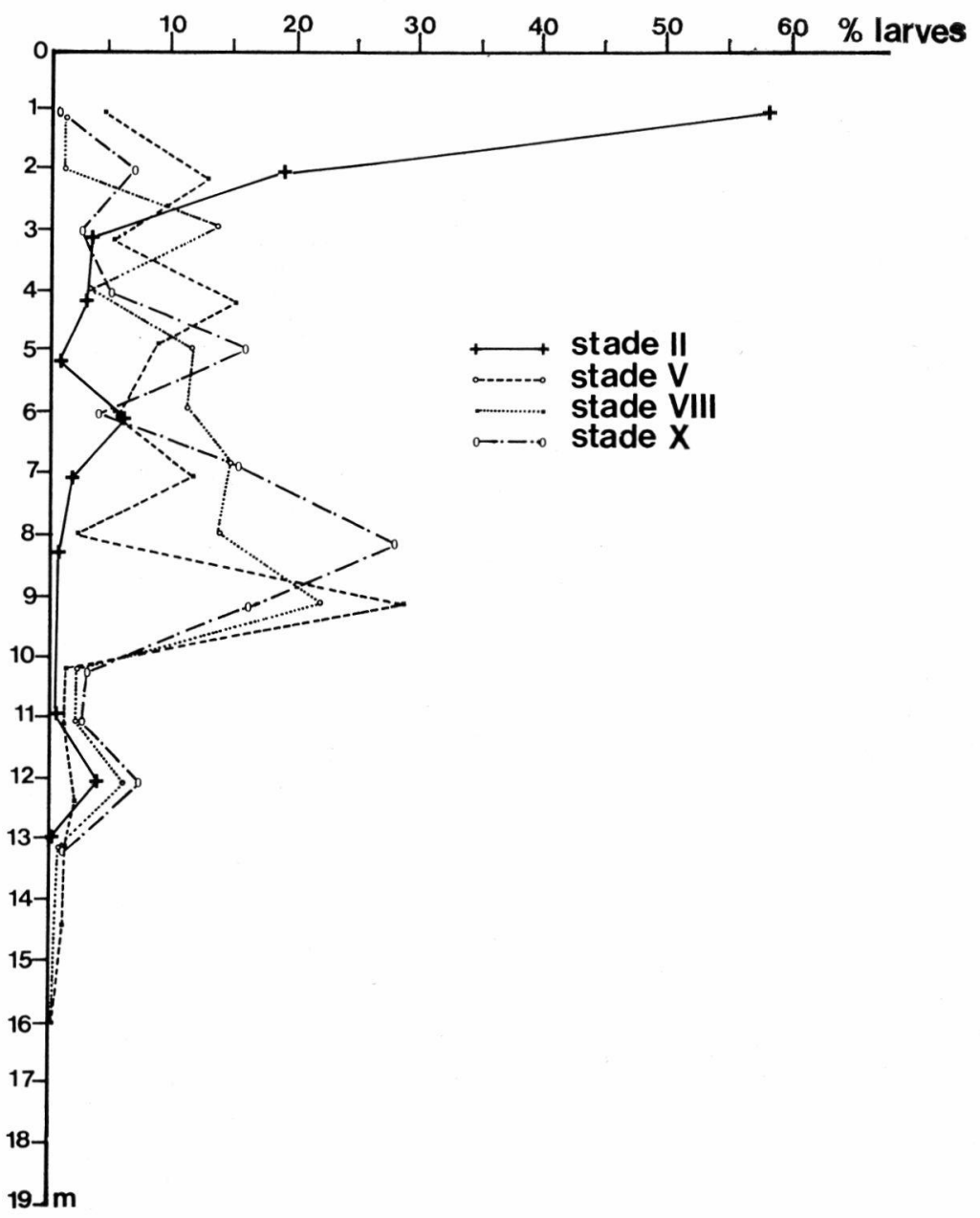

Fig. 4. - Répartition bathymétrique de 4 stades larvaires de Sialis lutaria.

La migration en profondeur peut être attribuée à une recherche de nourriture puisque la zone de plus forte densité de Sialis (7$9 \mathrm{~m}$ ) coïncide avec celle où le peuplement en invertébrés benthiques est le plus riche : 5142 ind./ $\mathrm{m}^{2}$ en moyenne (Laville 1971). C'est également, avec la zone littorale $(0-2 \mathrm{~m})$, la zone de densité optimum des Crustacés microbenthiques qui, comme nous le verrons ci-après, constituent une nourriture importante pour les larves de Sialis. 


\section{3. - NUTRITION DES LARVES}

Les larves de Sialis sont réputées prédatrices des organismes benthiques les plus abondants dans le milieu où elles vivent.

L'étude de leurs contenus stomacaux fournit des renseignements sur la qualité de la nourriture ingérée. Nous avons disséqué les tubes digestifs de nombreuses larves des différents stades mais le contenu stomacal d'un grand nombre d'individus s'est évéré impossible à identifier; nous récapitulons les résultats sur le tableau I.

Azam et Anderson (1969) pensent que la plus grande partie du développement du premier stade larvaire de deux Sialis (S. rotunda Banks et $S$. californica Banks) serait due à l'utilisation des réserves de nourriture stockées ou à l'ingestion de micro-organismes. De même, les larvules de $S$. lutaria se nourriraient de détritus et de micro-organismes (Dubois et Geigy 1935).

De jeunes larves de $S$. lutaria du Port-Bielh présentaient dans leur tractus digestif une larvule de Chironomide et un Ostracode, preuves d'une précoce activité prédatrice sur les invertébrés du benthos (tableau I).

Au cours de la première année, les Crustacés benthiques paraissent assurer l'essentiel de la nourriture; ce sont en premier lieu les Cladocères, puis dans l'ordre décroissant de leur importance au sein du benthos, les Cyclopides, les Ostracodes et enfin les Harpacticides qui sont les proies le plus souvent recensées dans les lubes digestifs des stades I à V. Chez les Cladocères, c'est la forme eurybathe et dominante Alona affinis Leydig qui est la plus fréquente dans le tractus des jeunes larves.

$\mathrm{Au}$ fur et à mesure du développement, la fréquence des larves et nymphes de Chironomides augmente; ils constituent la nourriture essentielle des trois derniers stades. Les onze espèces ou genres de Chironomides les plus fréquents du benthos ont été retrouvés comme proies à tous les stades de leur développement. Il n'apparaît pas de preferendum pour l'une ou l'autre espèce; la capture des proies s'effectue donc au hasard.

Des soies d'Oligochètes sont également visibles dans les tubes digestifs. Naididae et Tubificidae y sont fréquents du début à la fin du développement; ils jouent, sans aucun doute, un rôle important dans la nutrition des Sialis.

Les stades VII à $\mathrm{X}$ peuvent, en outre, exercer une prédation non négligeable sur les plus jeunes larves.

De nombreuses Diatomées (Pinnularia, Surrirella) sont souvent recensées chez certaines larves âgées; elles coïncident avec la présence de larves de Tanypodinae (Procladius, Ablabesmyia) qui s'en nourrissent plus ou moins abondamment. En effet, Morgan (1972) a pu estimer à $36 \%$ la quantité d'algues ingérées par des larves 
(9)

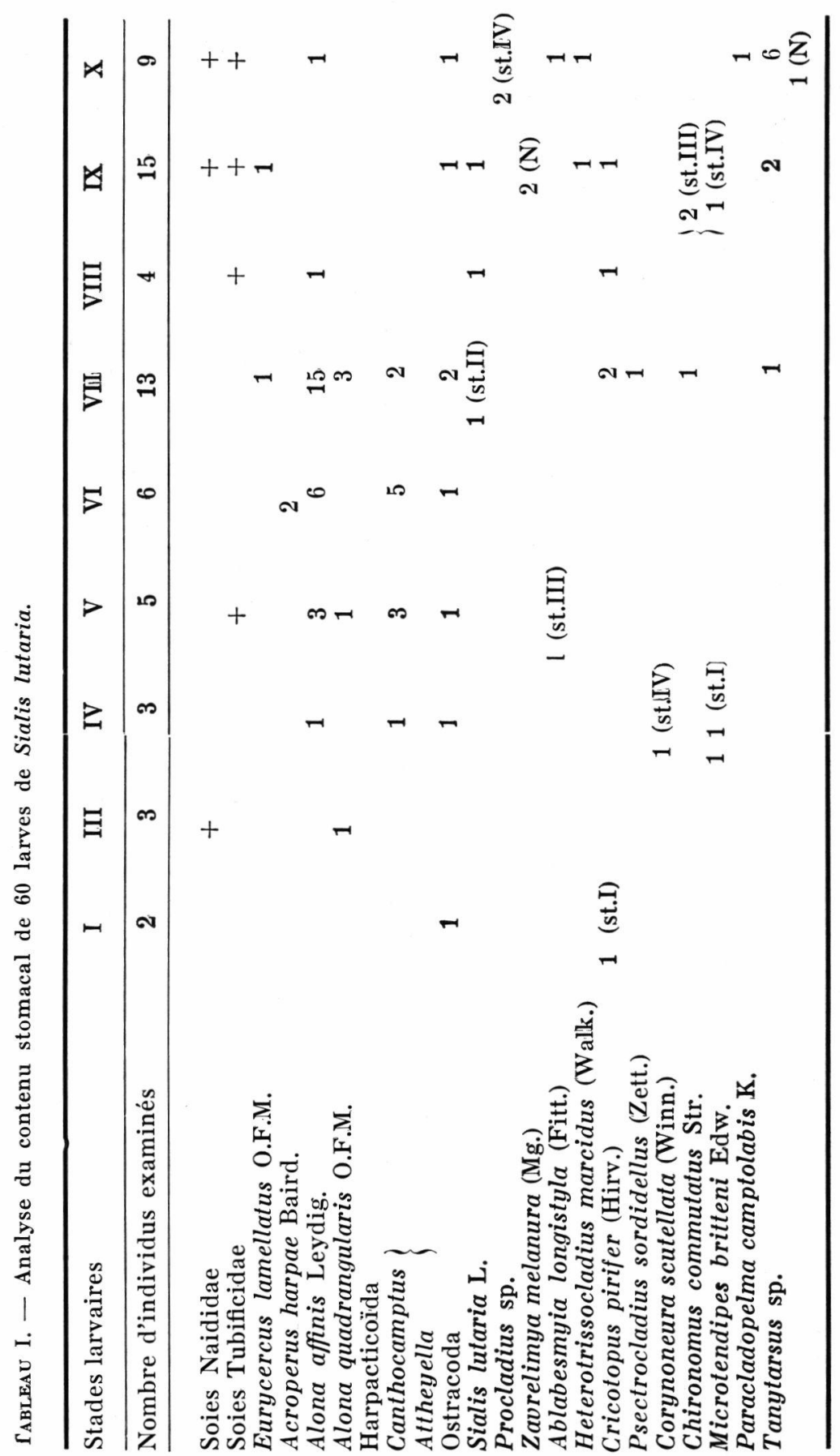


de Procladius, formes réputées carnivores, pour $28 \%$ seulement de proies animales. Selon Kajak et Dusoge (1970) ce mode de nutrition consisterait en un apport complémentaire à la nourriture d'origine animale des larves âgées.

En résumé, pendant la période de dégel, les Sialis exercent une prédation continue sur les autres représentants du macrobenthos essentiellement Chironomides (larves et nymphes) et Oligochètes ainsi que sur les Crustacés benthiques (Cladocères, Copépodes, Ostracodes). Ces derniers assurent l'essentiel de la nutrition des larves de première année.

\section{4. - PRODUCTION}

\section{1. - Evaluation de la densité moyenne du peuplement.}

Par suite des migrations des larves au cours de leur développement, nous avons calculé la densité moyenne des diverses cohortes dans la zone colonisée par Sialis lutaria $(0-15 \mathrm{~m})$. Les rares captures au-delà de cette profondeur n'ont pas été prises en compte.

Les récoltes, effectuées de janvier à octobre, ont permis de calculer l'abondance relative moyenne des stades II, V, VIII, X aux différentes profondeurs (fig. 4). Dans la zone littorale $(0-6,5 \mathrm{~m})$ et dans la partie supérieure de la zone «littori-profonde » $(6,5-$ $10 \mathrm{~m}$ ) la densité du peuplement est à peu près identique; par contre elle diminue nettement entre 10 et $15 \mathrm{~m}$.

La structure du peuplement et la densité des larves âgées de un, deux et trois ans dans chacune de ces zones permet ainsi d'évaluer à 194 larves $/ \mathrm{m}^{2}$ la densité moyenne de la population de S. lutaria entre $0 \mathrm{~m}$ et $15 \mathrm{~m}$ dont :

- 133 larves $(68 \%)$ au stade $\mathrm{V}$,

- 50 larves $(26 \%)$ au stade VIII,

- 11 larves $(6 \%)$ au stade $\mathrm{X}$ (tableau II).

\begin{tabular}{|c|c|c|c|c|c|}
\hline Stades & Densité $/ \mathrm{m}^{2}$ & Densité/m² & Densité $/ \mathrm{m}^{2}$ & $\begin{array}{l}\text { Densité } \\
\text { moyenne/m² }\end{array}$ & $\begin{array}{l}\text { Abondance } \\
\text { relative }\end{array}$ \\
\hline larvaires & $0-6,5 \mathrm{~m}$ & $6,5-10$ & $10-15$ & $0.15 \mathrm{~m}$ & moyenne $\%$ \\
\hline $\mathbf{V}$ & 199 & 175 & 24 & 133 & 68 \\
\hline VIII & 66 & 76 & 8 & 50 & 26 \\
\hline \multirow[t]{2}{*}{$\mathbf{X}$} & 12 & 19 & 3 & 11 & 6 \\
\hline & 277 & 270 & 35 & 194 & 100 \\
\hline
\end{tabular}

TABleau II. - Densité et abondance relatives des stades larvaires de un (V), deux (VIII) et trois ans (X) dans trois zones du lac de Port-Bielh au dégel. 


\section{2. - Croissance pondérale.}

Une difficulté fondamentale dans les calculs de production est d'obtenir des données valables de la durée du développement et de la croissance des organismes, particulièrement chez les espèces présentant une longue période de reproduction.

Aussi, après avoir mis en évidence le cycle biologique de Sialis lutaria, nous nous sommes attachés à estimer la croissance larvaire au cours de son développement.

13 lots, chacun de 5 à 21 larves longues de 3 à $18 \mathrm{~mm}$ (soie terminale du $10^{\circ}$ segment exclue) et groupées par classe de taille de $2 \mathrm{~mm}$, ont été pesés après évaporation de l'eau superficielle (PF) et après séchage pendant 18 heures environ dans une étuve à $105{ }^{\circ} \mathrm{C}$ (PS).

Les poids secs moyens des différentes classes de taille ont été portés en fonction de la taille dans la figure 5 en coordonnées logarithmiques. La droite de régression $(\log \mathrm{PS}=2,952 \log \mathrm{L}$ - 0,559) permet d'évaluer le poids moyen d'une larve au cours de son développement.

L'évolution pondérale moyenne d'une larve pendant son cycle est tracée sur la figure 6; elle met en évidence quatre périodes de forte croissance séparées par trois périodes de croissance ralentie voire d'arrêt de croissance.

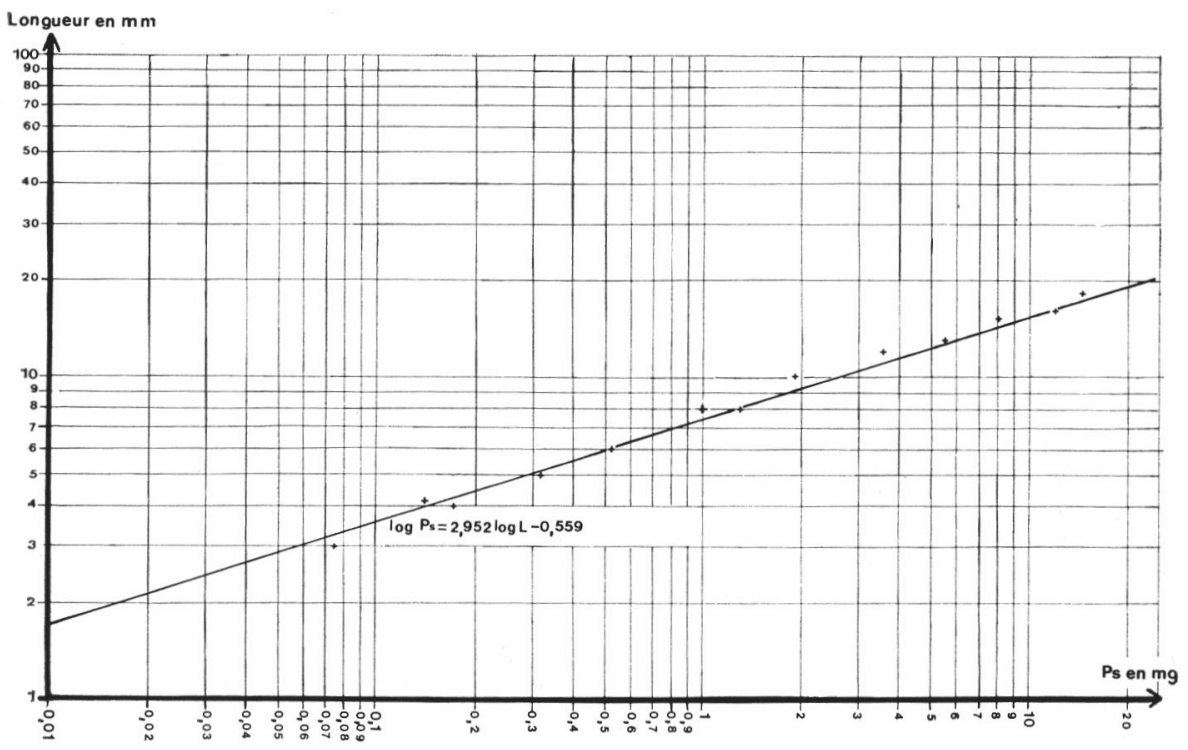

FIG. 5. - Relation taille - poids sec chez les larves de Sialis lutaria. 


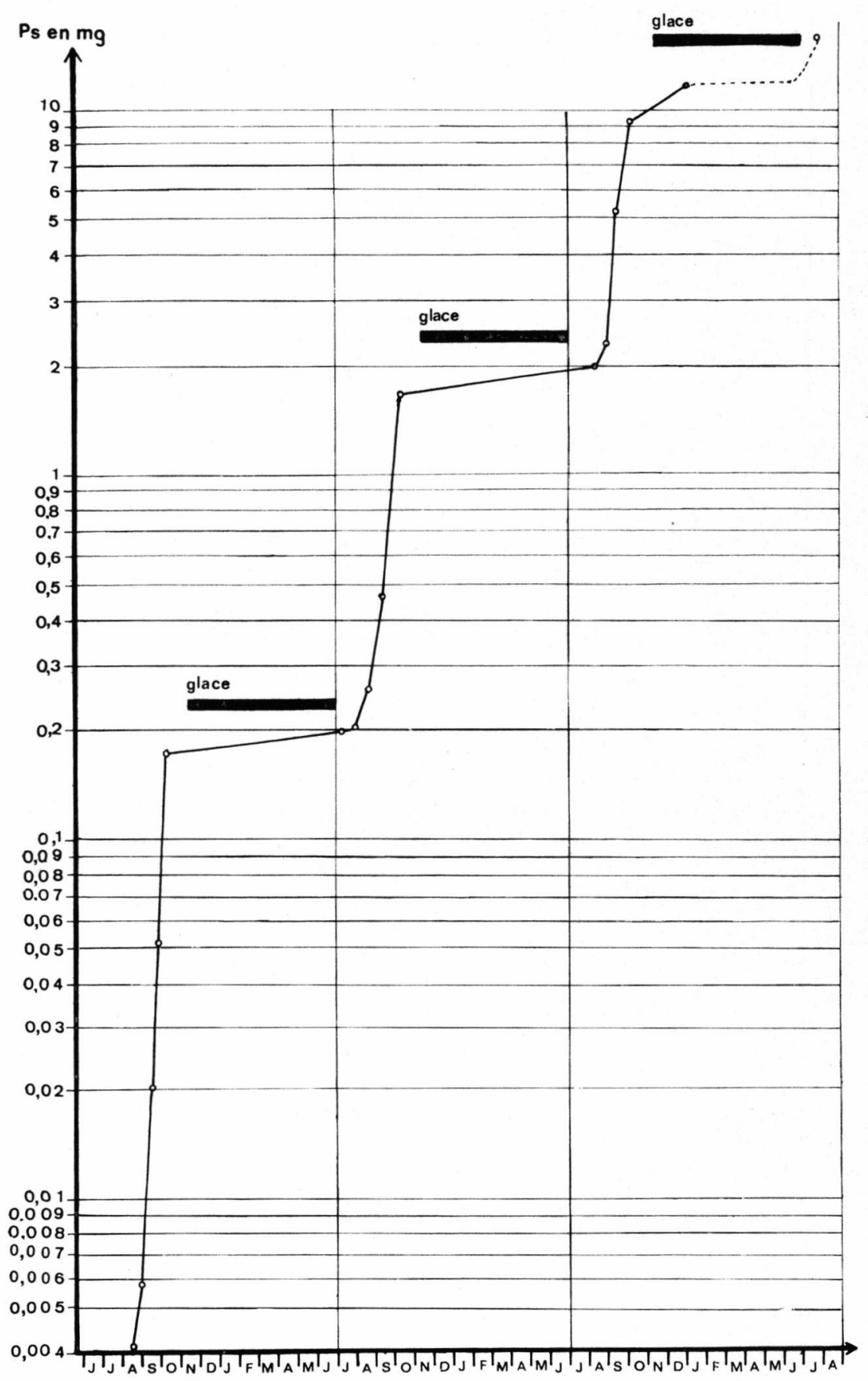

FIG. 6. - Variation du poids sec moyen de la larve de Sialis lutaria au cours de son développement. 
La première période de croissance marque le passage de la larve au stade $\mathrm{V}$; son poids, à la mi-octobre, atteint $0,18 \mathrm{mg}$ et avoisine $0,20 \mathrm{mg}$ au dégel.

La deuxième période de croissance correspond au passage au stade VIII de la larve; son poids, à la mi-octobre, est de $1,7 \mathrm{mg}$; il atteindra $2 \mathrm{mg}$ au dégel.

Au cours du troisième été, la larve effectue ses deux dernières mues; elle atteint $9 \mathrm{mg}$ vers la mi-octobre et $12 \mathrm{mg}$ au mois de décembre. Lors de cette période se situe l'accroissement le plus important du cycle; il est égal à $66,7 \%$ du poids final. La larve de décembre atteint $80 \%$ de son poids définitif.

Il est probable que la croissance se ralentit entre décembre et juin et que les larves terminent leur croissance au dégel pendant le mois de juillet du quatrième été, pour atteindre $15 \mathrm{mg}$ avant la nymphose.

Tout comme chez le Chironomide Chironomus commutatus Str. qui, lui, met deux années pour accomplir son développement (Laville 1971), les périodes d'accroissement maximum interviennent au moment de l'isothermie d'automne qui, suivant les années, peut se prolonger jusqu'à fin novembre. La croissance hivernale, très ralentie, est peu importante.

\section{3. - Calculs de la production.}

A partir des données de base, cycle de développement, accroissement en poids, et densité moyenne des trois cohortes au dégel, nous avons calculé la production annuelle nette (P) des Sialis du lac de Port-Bielh.

A cet effet nous avons utilisé la formule dérivée de celle de Boysen - Jensen (1919) appropriée, selon Kajak (1967), pour des calculs de production basés sur les variations d'abondance et de biomasse :

$$
\mathrm{P}=\mathrm{N} 2 .(\mathrm{W} 2-\mathrm{W} 1)+\mathrm{N} 1-\mathrm{N} 2 \frac{\mathrm{W} 2-\mathrm{W} 1}{2}
$$

$\mathrm{P}=$ production pour la période $\mathrm{t} 2-\mathrm{t} 1 ; \mathrm{N} 1$ et $\mathrm{N} 2=$ nombres d'individus anx temps $\mathrm{t} 1$ et $\mathrm{t} 2$; $\mathrm{W} 1$ et $\mathrm{W} 2=$ poids sec d'un individu aux temps $\mathrm{t} 1$ et $\mathrm{t} 2$.

La première partie de la formule permet d'obtenir la production annuelle de chacune des trois cohortes, connaissant l'augmentation du poids d'un individu pendant une année. La production des animaux morts dans cet intervalle est donnée par la deuxième partie de la formule; elle s'obtient en multipliant l'accroissement moyen par le nombre d'individus disparus pendant l'année. 
N. GIANI ET H. LAVILLE

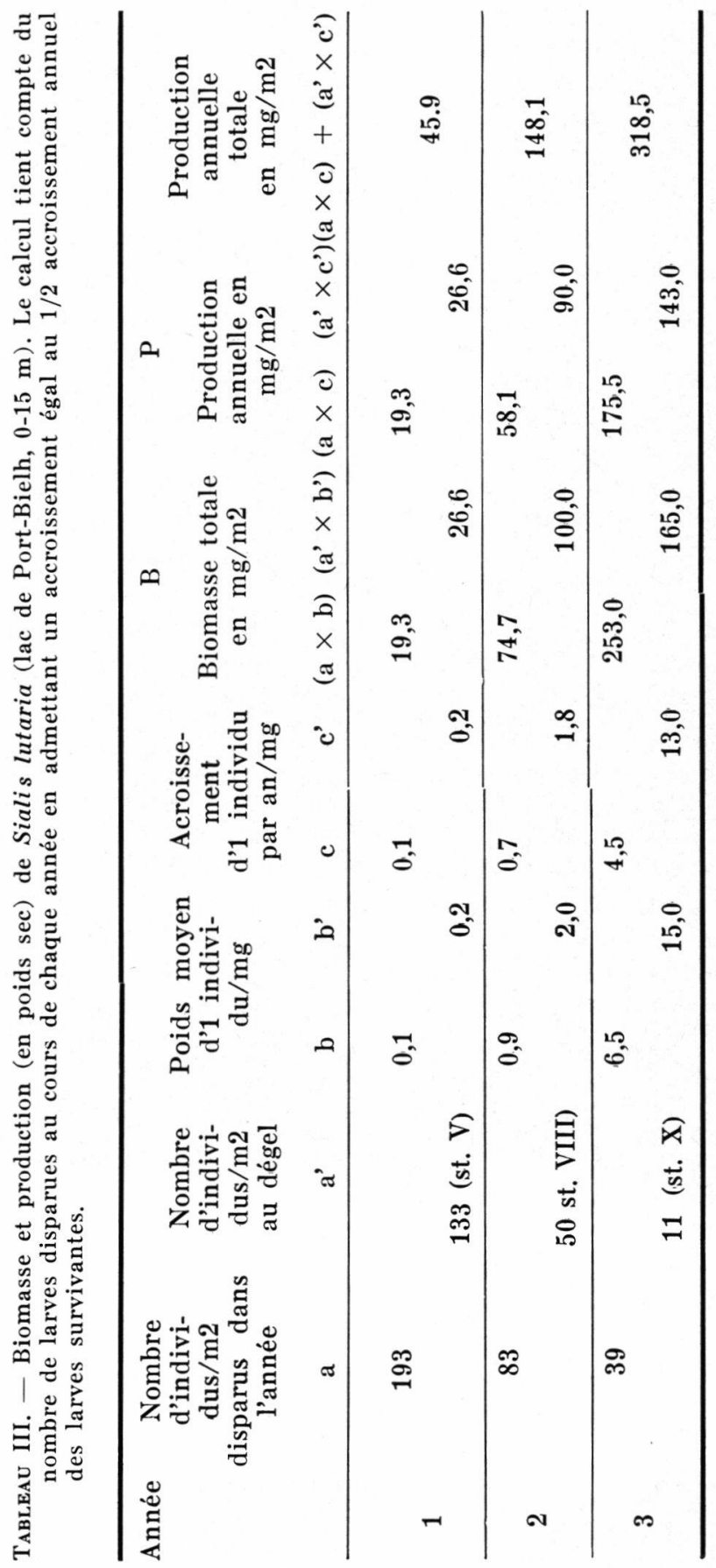


La production annuelle des trois cohortes est résumée dans le tableau III. L'estimation du nombre de larvules au terme des éclosions (fin août) a été faite en admettant que la courbe de mortalité larvaire entre l'éclosion et la nymphose avait la forme d'une exponentielle décroissante. On aurait ainsi 326 larves $/ \mathrm{m}^{2}$ au mois d'août dont 194 seulement atteindraient le stade V. La production correspondante représente à peine $3,5 \%$ de la production totale. L'erreur commise en négligeant ces premiers stades dont la densité est, par ailleurs, très difficile à évaluer, s'avère donc relativement faible. La production des animaux disparus au cours de la deuxième année représente $11,4 \%$, celle des larves mortes pendant la troisième année atteint plus du tiers de la production totale $(34,35 \%)$. C'est, en fait, au cours de la troisième année du développement que s'effectuent près des deux tiers de la production des Sialis $(62,15 \%)$, le tiers restant se répartisssant entre la première $(8,95 \%)$ et la deuxième année $(28,90 \%)$.

Avec des densités moyennes de l'ordre de 46 à 291 individus par $\mathrm{m}^{2}$, la production annuelle de Sialis lutaria au lac de PortBielh $(5,12 \mathrm{~kg} / \mathrm{ha})$ atteint 1,9 fois celle de Zavrelimyia melanura (Mg.) $(2,7 \mathrm{~kg} / \mathrm{ha})$ et plus des deux tiers $(67 \%)$ de la production du Chironomide dominant de ce lac Psectrocladius sordidellus (Zett.) $(7,6 \mathrm{~kg} / \mathrm{ha})$ (Laville 1971$)$.

Comme pour les Chironomides, la biomasse maximale des larves de $S$. lutaria se situe vers la fin du moins de novembre, juste avant la période de gel. On peut alors l'estimer à $382 \mathrm{mg} / \mathrm{m}^{2}$, soit un rapport $P / B$ max. de 1,34 . Cette valeur n'est pas très éloignée des valeurs obtenues pour les deux Chironomides univoltins $Z$. melanura $(1,5)$ et Ps. sordidellus $(1,65)$ de ce lac. Ce résultat tend à confirmer une certaine stabilité de ce facteur, même pour des insectes à cycle de développement plus long; il permet en outre d'envisager sa généralisation (par exemple à un taux moyen de 1,5) pour obtenir l'évaluation rapide de la production nette des insectes benthiques des lacs de haute montagne, après simple évaluation de leur biomasse maximale à l'entrée de l'hiver.

\section{Rapport PS/PF}

Le facteur de conversion couramment utilisé pour obtenir des valeurs de biomasses ou de productions en poids frais (PF) à partir des pesées, plus précises, en poids sec (PS) est, pour les invertébrés du benthos $\mathrm{PS} / \mathrm{PF}=15 \%$ (Kajak 1967).

Les pesées de 13 lots de larves avant et après passage à l'étuve ont montré que si ce rapport était bien de $15 \%$ pour les larves de 5 à $7 \mathrm{~mm}$ de long (stades V-VII), il atteignait 17,5\% pour celles de 8 à $14 \mathrm{~mm}$ (stades VIII - IX) et 24,5\% pour celles 
comprises entre 15 et $18 \mathrm{~mm}$ (stade X). Cette augmentation correspond à l'accroissement relatif des parties chitineuses de la larve au cours du développement.

Si on applique ces trois différents facteurs aux diverses cohortes nous évaluons la production en poids frais des Sialis du Port-Bielh à $2794,34 \mathrm{mg} / \mathrm{m}^{2}$ soit $27,94 \mathrm{~kg} / \mathrm{ha} / \mathrm{an}$.

En utilisant le même facteur de $15 \%$ on obtient une production en $\mathrm{PF}$ égale à $35,08 \mathrm{~kg} / \mathrm{ha} / \mathrm{an}$. Ce résultat surévalue de $22,7 \%$ la valeur de la production précédente.

\section{5. - IMPORTANCE DES SIALIS DANS L'ÉCOSYSTEME}

L'analyse de quelques contenus stomacaux de truites (Salmo trutta fario L.) capturées au dégel, le 2 juillet 1971 fait apparaître l'importance des Sialis dans leur nutrition (tableau IV). Toutes les larves ingérées sont aux deux derniers stades du développement. Les autres proies recensées en même temps sont des nymphes du Tanytarsini Micropsectra contracta Reiss, Chironomide surtout abondant entre 0 et $5 \mathrm{~m}$ et dont les émergences précoces sont localisées, à cette époque, dans la zone comprise entre 0 et $3 \mathrm{~m}$. Il semble donc qu'au dégel, les truites se nourrissent au bord, essentiellement à partir des larves âgées de Sialis au moment de leur migration vers le littoral.

Tableau IV. - Analyse du contenu stomacal de 7 truites (Salmo trutta fario) capturées le 2 juillet 1971; (L) = larves, $(\mathrm{N})=$ Nymphes.

\begin{tabular}{|c|c|c|c|c|c|c|c|}
\hline \multirow[t]{2}{*}{ Proies } & \multicolumn{7}{|c|}{ Salmo trutta fario L.: longueur $/ \mathrm{mm}$} \\
\hline & 190 & 212 & 235 & 274 & 282 & 286 & $\mathbf{3 1 0}$ \\
\hline Sialis lutaria (L) & 3 & 58 & 54 & 87 & 58 & 94 & 118 \\
\hline Ephémères (L) & 2 & & & 5 & & & \\
\hline Trichoptères (L) & 1 & & & 1 & & & \\
\hline Micropsectra contracta $(\mathrm{N})$ & & 127 & & 99 & 1 & 6 & \\
\hline Zavrelimyia melanura (L) & 108 & & & 2 & & & \\
\hline Psectrocladius sordidellus (L) & 1 & & & $\mathbf{1}$ & & & \\
\hline Heterotrissocladius marcidus (L) & & & & 1 & & & \\
\hline
\end{tabular}

Il est probable que la prédation des truites persiste pendant l'hiver. Les émergences de Chironomides terminées, les proies alors disponibles sont, avant tout, les larves libres de Sialis et de Tanypodinae.

Nous n'avons pas de données se rapportant à la nutrition des truites pendant I'hiver, mais d'après celles de Elliott et Jenkins (1972) elle n'est pas négligeable même lorsque la température avoisine $1{ }^{\circ} \mathrm{C}$. La nutrition dépendrait alors davantage de la disponibilité de la nourriture que d'un changement dans l'activité de la truite ou dans son empressement à se nourrir. 
Berglund (1968) signale de son côté que la plus forte prédation de Salmo trutta coïncide avec le maximum d'Asellus disponible sous la glace au début du printemps.

Tant par sa production que par sa position trophique, Sialis lutaria joue un rôle important au sein du lac de Port-Bielh. Son mode de nutrition carnivore en fait notamment l'intermédiaire entre les principaux représentants $\mathrm{du}$ benthos (Crustacés, Chironomides, Oligochètes) et la truite qui occupe l'extrémité de la chaîne alimentaire.

\section{TRAVAOX CITES}

Azam (K.M.) and ANderson (N.H.). 1969. - Life history and habits of Sialis rotunda and Sialis californica in Western Oregon. Ann. ent. Soc. Am., 62 (3) : 549-558.

BERGLUND (T.). 1969. - The influence of predation by brown trout on Asellus in a pond. Rep. Inst. Freshw. Res. Drottningholm, 48 : 77-101.

Bertrand (H.). 1954. - Les insectes aquatiques d'Europe (vol. I). Encyclopédie entomologique, Série A, XXX Lechevalier, Paris : $556 \mathrm{p}$.

Boysen-JEnsen (P.). - Valuation of the Limfjord. I. Studies on the fish food on the Limfjord 1909-1917. Rep. Dan. biol. Stn., 26 : 1-44.

Dubors und Geigy (R.). 1935. - Beiträge zur ökologie, Fortpflanzungsbiologie und Metamorphose von Sialis lutaria L. Rev. Suisse Zool., 43 : 519-523.

Flliote (G.V.) and Jenkins J.R. (T.M.). 1972. - Winter food of trout in three high elevation Sierra Nevada lakes. Calif. Fish and Game, 58 (3) : 231-237.

KaJAK (Z.). 1967. - Remarques sur les méthodes d'investigations de la production du benthos (Polonais avec résumé anglais). Ekol. Pol. Sér. B., XIIII (2) : 173-195.

KaJak (Z.) and Dusoge (K.). 1970. - Production efficiency of Procladius choreus. Mg. (Chironomidae, Diptera) and its dependance on the trophic conditions Pol. Arch. Hydrobiol., 17 (30) : 217-224.

LAville (H.) . 1971. - Recherches sur Ies Chironomides (Diptera) lacustres du massif de Néouvielle (Hautes-Pyrénées). Deuxième partie : Communautés et Production. Annls limnol., 7 (3) : 335-414.

Lestage (J. A.). 1918. - Notes biologiques sur Sialis lutaria L. (Megaloptera). Ann. Biol. lacustre, $9: 25-38$.

(J.A.). 1920. - Le mécanisme de la ponte chez Sialis lutaria (L.) (Megaloptera). Ann. Biol. lacustre, 10: 221-223.

Macan (T.T.) and Worthington (E.B.). 1968. - Life in lakes and Rivers (second edition). Collins, London : $272 \mathrm{p}$.

Morgan (N.C.). 1972. - Productivity studies at Loch Leven (a shallow nutrient rich lowland lake). Proceedings of the IBP-UNESCO. Symposium on Productivity problems of freshwaters, Kazimierz Poland, May 6-12, 1970, Editors : Z. Kajak, A. Hillbricht-Ilkowska : 183-205.

Thienemann (A.). 1925. - Die Binnengewässer Mitteleuropas. Eine limnogische Einführung. Die Binnengewässer 1. Stuttgart : $255 \mathrm{p}$. 